\title{
Chinese SLE Treatment and Research group (CSTAR) registry 2009-2019: Major clinical characteristics of Chinese patients with systemic lupus erythematosus
}

\author{
Mengtao Li', Yanhong Wang ${ }^{2}$, Jiuliang Zhao', Qian Wang', Ziqian Wang'1, Xinping Tian'1, Xiaofeng Zeng', ${ }^{1, *}$ \\ ${ }^{1}$ Department of Rheumatology, Peking Union Medical College Hospital (PUMCH), Chinese Academy of Medical Sciences, National \\ Clinical Research Center for Dermatologic and Immunologic Diseases (NCRC-DID), Key Laboratory of Rheumatology and Clinical \\ Immunology, Ministry of Education, Chinese Rheumatism Data Center (CRDC), Chinese SLE Treatment and Research Group \\ (CSTAR), Beijing, China of Rheumatology, Peking Union Medical College Hospital, Beijing, China \\ ${ }^{2}$ Department of Epidemiology and Biostatistics, Peking Union Medical College, Beijing, China
}

Abstract

Received December 29, 2020 accepted February 4, 2021

Objective: To describe the overall clinical characteristics of patients from the Chinese SLE Treatment and Research group (CSTAR) registry in the past 10 years.

Methods: CSTAR registry originated as a multicenter, consecutive, and prospective design launched in 2009. The data were collected online from 304 rheumatology centers, which covered 30 provinces in China. All data were generated and uploaded in the clinic directly without secondary collection, including demographic, clinical manifestations, disease activity (SLEDAI-2K) and organ damage evaluation (SLICC Damage Index), and lab test results. Biological samples were preserved for future study. Meanwhile, data cleaning and validation were managed by a professional backstage statistician.

Results: A total of 25,147 SLE patients were registered up to Dec 2019. The mean age of disease onset was 31.2 years with the age of confirmed diagnosis at 32.1 years. The male to female rate was $1: 11.9 .4 .6 \%$ were pediatric patients. The most common clinical presentations at entry were oral ulcer $(59.4 \%)$, arthritis $(55.0 \%)$, alopecia $(43.22 \%)$, skin rash $(40.0 \%)$, and nephritis (33.5\%). The mean SLEDAl score at entry was 4 and $32.6 \%$ were in moderate to severely active disease. $\mathbf{6 6 . 4 \%}$ and $\mathbf{3 7 . 8 \%}$ of patients were positive for anti-ds-DNA antibody or low complement level. Additionally, $\mathbf{1 . 1 \%}$ of patients were with pulmonary arterial hypertension (PAH). The prevalence rate of cerebrovascular disease was $0.3 \%$. A total of $58.2 \%$ of patients were in clinical remission when thery were registered.

Conclusions: The CSTAR registry is the largest ongoing SLE registry in China so far. More than 25,000 SLE patients are registered and nearly 10,000 are in follow-up visits. This registry has provided high-quality data for future studies and will become an infrastructure for domestic and international collaborations.

Keywords

systemic lupus erythematosus $\cdot$ registry $\cdot$ CSTAR $\cdot$ epidemiology

\section{Introduction}

Systemic lupus erythematosus (SLE) is a complex systemic autoimmune disease that could lead to multi-system involvement, irreversible multi-organ damage, and eventually lead to death. The disease is characterized by repeated flares and remissions, and the presence of autoantibodies in the blood. The prevalence of SLE varies across the world ranging from $0 \sim 241 / 100,000$ globally. It is estimated that the prevalence of SLE is 30 70/100,000 in mainland China, ${ }^{[1,2]}$ most of them are women.

With the continuous improvement of the diagnosis and treatment of SLE, the survival rate has greatly improved. The 5 -year survival rate of SLE patients increased from $50 \%$ to $60 \%$ in the 1950 s to over $90 \%$ in the 1990 s and has gradually stabilized from 2008 to $2016 \cdot{ }^{[3-5]}$ SLE has changed from an acute fatal disease to a chronic controllable disease.

Lupus registries have been created and maintained worldwide for better understanding and management of this chronic disease, such as the Systemic Lupus International Collaborative Clinics (SLICC), ${ }^{[6]}$ Lupus Family Registry and Repository (LFRR), ${ }^{[7]}$ Lupus in Minorities: Nature vs Nurture (LUMINA) ${ }^{[8]}$ Euro-lupus project,,${ }^{[9]}$ John Hopkins, ${ }^{[10]}$ the PROFILEE ${ }^{[11]}$ and the GLADEL cohorts. ${ }^{[12]}$ The data and 
evidence from these registries have guided clinical practice to improve the prognosis and quality of life of SLE patients.

There is no large-scale registry for SLE in China although the development of rheumatology in China is rapid in the past 20 years. The Chinese SLE Treatment and Research group (CSTAR) initiated the first online registry of Chinese lupus in 2009 under the support of the Chinese National Key Technology R\&D Program, which was developed as a multicenter observational cohort initially in 2009 attempting to depict major clinical characteristics of lupus in Chinese patients. This article introduces the major clinical characteristics of Chinese SLE patients enrolled in this registry from 2009 to 2019.

\section{Methods}

\section{CSTAR}

CSTAR is a national liaison led by Peking Union Medical College Hospital (PUMCH) that was first funded by the Chinese Ministry of Science \& Technology in 2009. By the end of 2019, 309 high-ranked rheumatology centers (see appendix) in total from 30 provinces in China joined the registry. The missions of CSTAR are: 1 . to explore the epidemiological and clinical pattern of Chinese SLE patients, 2. to provide the infrastructure for conducting clinical and basic research on SLE with coordinated nationwide efforts, 3 . to set up a nation-wide union for Chinese SLE research force, and 4 . to create a self-sustaining research model.

\section{Registry}

The first project launched by CSTAR in 2009 was the registry for Chinese lupus patients, which was approved by the Institute Review Board (IRB) of PUMCH. Other centers had received their own ethical approval by the local IRBs according to local regulations. All investigators were trained for diagnosis confirmation, history review, disease activity evaluation, mandatory laboratory tests, data input, and biologic sample collections through national or regional training programs. Chinese SLE information system (CSIS), which is the official CSTAR registry online system, is open to qualified investigators from all sites. In addition, newsletters of enrollment numbers, preliminary data, and quality controls are sent to all centers every month.

\section{Patient Recruitment}

SLE patients were registered if they fulfilled the SLE classification criteria revised by the American College of Rheumatology (ACR) in 1997, ${ }^{[13]}$ or 2012 SLICC classification criteria, ${ }^{[14]}$ or the 2019 EULAR classification criteria, ${ }^{[15]}$ and signed the informed consent form by themselves or their authorized guardians. This ongoing registry was first launched in April 2009 and had recruited 25,147 SLE patients by December 2019. In this study, we analyzed baseline data of 25,147 SLE patients based on the CSTAR online registry.

\section{Data Collection}

All CSTAR centers use the same protocol and operation procedures for data collection. Data collected including demographic data, age at entry, age at disease onset, age at diagnosis, family history of rheumatic diseases, reproductive history, as well as socioeconomic status, education, and marital status.

Clinical features and organ involvement were collected, evaluated, and the relevant data were entered into the online CSTAR registry database. Clinical manifestations included in this registry were rash (acute or subacute rashes, chronic skin rashes, and lupus profundus), ulcers (oral or nasal ulcer), non-scarring hair loss, musculoskeletal involvement (arthritis and myositis), hematologic involvement (anemia, leukopenia, thrombocytopenia, and thrombotic thrombocytopenic purpura), serositis, renal involvement, nephropathy, and neurologic involvement. Some rare complications such as pulmonary arterial hypertension (PAH) and interstitial lung disease (ILD) were also collected.

The levels of autoantibodies were measured at the local laboratories of each center and included anti-nuclear antibody (ANA), anti-double strand DNA (anti-dsDNA) antibody, anti-Sm antibody, anti-SSA antibody, anti-SSB, anti-u1 small-nuclear RNA-protein (anti-RNP) antibody, and anti-ribosomal RNA-protein (anti-rRNP). Most centers tested ANA and anti-dsDNA antibody by immunofluorescence assay with Hep-2 cell line and the anti-extractable nuclear antigen (ENA) antibody (including anti-Sm, anti-SSA, anti-SSB, anti-RNP, and anti-rRNP antibodies) were tested with the immunoblotting assay. Anti-phospholipid antibodies (aPLs) were not mandatory tests unless antiphospholipid syndrome was suspected. Complement levels were also tested and recorded in the registry.

Disease activity was evaluated in all SLE patients by the SLE disease activity index (SLEDAI-2000) ${ }^{[16]}$ and physician global assessment (PGA).

\section{Statistical Analysis}

Baseline data were analyzed using SASP software. Variables were described using counts and/or percentages or medians and ranges. 


\section{Results}

\section{Demographics}

The baseline data of 25,147 SLE patients ' were analyzed in total. 23,201 (92.3\%) patients were female and 1946 (7.7\%) patients were male, with the female-to-male ratio of 11.9:1. $1152(4.6 \%)$ patients were aged 5-17 years old and the female-to-male ratio was $6.2: 1$ in this group of patients. The mean age at entry was $36.1 \pm 12.9$-year-old and the mean age at diagnosis of lupus was $32.1 \pm 12$.8-year-old. The mean age at onset of initial symptoms was $31.2 \pm 12.6$ years. Comorbid conditions presented less than one percent of the total SLE patients, including coronary heart disease $(0.9 \%)$, stroke $(0.8 \%)$, fragile fracture $(0.8 \%)$, and cancer $(0.7 \%) .777$ (3.6\%) patients were pregnant at entry and 3002 (19.3\%) patients were dysgenesis at baseline.

\section{Clinical Manifestations}

The baseline data of 25,147 patients showed that 14,948 $(59.4 \%)$ patients presented with skin and mucosa lesions, $13,842(55.0 \%)$ patients had arthritis, 10,868 (43.2\%) patients had alopecia, 10,066 (40.0\%) patients presented with acute and subacute cutaneous lupus, 8430 (33.5\%) had kidney involvement, $7028(28.0 \%)$ presented with chronic skin lesions and lupus profundus, 5876 (23.4\%) patients had leukopenia or lymphopenia, 5710 (22.7\%) patients had ulcers, 4352 $(17.3 \%)$ had thrombocytopenia, and 2785 (11.1\%) patients had serositis. In addition, $1.1 \%$ of patients had confirmed $\mathrm{PAH}$ and the prevalence rate of cerebrovascular disease was $0.3 \%$. More than half of Chinese SLE patients $(55.14 \%)$ presented with musculoskeletal involvement, $37.2 \%$ of patients had hematological disorders and other systems were presented in a much lower frequency such as neuropathy (1.5\%), gastrointestinal involvement(1.0\%), cardiopulmonary abnormalities(2.5\%), and ophthalmological lesions (0.3\%).

\section{Autoantibodies and Complement Level}

The most common autoantibodies were positive ANA, which was positive in 23,777 (94.6\%) patients, the anti-dsDNA antibody was positive in $16,704(66.4 \%)$ patients, the antiSm antibody was positive in 9506 (37.8\%) patients. Low complement (Low C3 or C4) level was presented in 9721 $(38.7 \%)$ patients at entry.

\section{Disease Activity}

Every patient was assessed by SLEDAI and PGA for disease activity when being registered. The median SLEDAI score was 4 (IQR 9) and the median PGA was 1.1 (IQR 1.0).
The patients were stratified by SLEDAI scores into groups, including $16,705(66.4 \%)$ patients in the mild active group (£6), $4780(19.0 \%)$ patients in the moderately active group $(7-12)$, and $3660(14.6 \%)$ patients in the very active group (>12). In addition, 14,641 (58.2\%) patients had SLEDAI score $\leq 4$ at registration, which means over half of the CSTAR registry patients were in clinical remission when they were registered. However, more than $40 \%$ of patients remained active during the follow-up.

\section{Discussion}

SLE has been recognized as a chronic, life-threatening rheumatic disease with strong heterogeneity and requires individualized diagnosis and treatment. It is well known that SLE affects women more often than men and is much more prevalent in certain ethnic groups, such as African-Americans and Asians. ${ }^{[17]}$ The prevalence of SLE in Chinese ranks the second in the world,i.e., more prevalent than Caucasians but less than African-Americans. ${ }^{[1,2]}$ It is estimated that the total number of SLE patients in China mainland is more than one million. However, most studies in the past were retrospective or conducted in a relatively patient population. ${ }^{[18,19]}$

As the first national lupus study group in China, CSTAR initiated an online SLE registry in 2009. The CSTAR registry now has become the largest prospective SLE cohort in China. The number of participating centers has increased from 104 provincial hospitals in 2009 to 309 hospitals in 2019, which has covered most of China's mainland. More than $85 \%$ of patients registered in recent 4 years and more than $40 \%$ of all registered SLE patients had follow-up records. These data show the improved awareness of disease management in China from both physicians' and patients' perspectives. With strict quality control measures and protocol-directed data collection operation, the CSTAR registry can provide high quality and reliable data of Chinese SLE patients for a better understanding of the disease pattern as well as for conducting research projects. Early in this year, findings from the CSTAR registry have served as solid evidence for the 2020 Chinese guidelines for the diagnosis and treatment of systemic lupus erythematosus. ${ }^{[20]}$

As data in the CSTAR registry has shown, SLE mainly occurred in childbearing women which is similar to and consistent with other studies. It is worth pointing out that patients in the CSTAR registry were diagnosed at an average age of 32.1 years old, which is less than 1 year after the onset of the disease symptoms(31.2 years). This suggests that along with the development of rheumatology in China, both the awareness of the disease and diagnosis assessments 
were much improved in the past 10 years. Residential region records have shown that in the CSTAR registry, more patients are from the northeast (8.3\%) and northwest $(7.9 \%)$ part of China, but much less from the northern $(25.0 \%)$ or eastern $(20.3 \%)$ part of China. This may be caused by the unbalance of medical resources in China. To promote the set-up and development of rheumatology in general hospitals, and to improve the diagnosis and treatment capacity for rheumatic diseases of the country, the National Health Commission of the PRC had released a guideline for the set-up and management of the rheumatology department in general hospitals last year. In the CSTAR registry, approximately $20 \%$ of the total patient population could not get pregnant. This is in accordance with other studies. This implies that a greater risk of dysgenesis in SLE patients than in the general population. ${ }^{[21]}$ Pre-conception counseling for patients with SLE is critical to a successful pregnancy. A planned pregnancy could significantly reduce the risk of flares during pregnancy and improve pregnancy outcomes. ${ }^{[22,23]}$

In the CSTAR registry, the most common clinical manifestations are skin and mucosal lesions, arthritis, hair loss, and kidney involvement. This disease patterns is similar with other studies. In addition, some clinical manifestations have been shown a downward tendency compared with a few years ago. For example, the percentage of kidney involvement in 2104 SLE patients registered in the CSTAR was $47.4 \%$ in 2010 , but this has decreased to $33.5 \%$ in this study. ${ }^{[24]}$

Autoantibodies and complement levels were assessed in local laboratories according to the CSTAR registry guideline. However, without the confirmation of antibodies by a central lab, we could not analyze the pattern of phenotypes associated with these antibodies. From current results, the most common antibody that is positive in SLE patients is ANA, followed by anti-ds-DNA antibody in $66.4 \%$ of patients. Approximately $40 \%$ of patients had low blood complement levels and $79 \%$ of patients had either positive anti-ds-DNA or low complement when being registered. According to the CSTAR registry protocol, all DNA and serum samples will be transported to the leading site, $\mathrm{PUMCH}$, for future analysis.

In the newly released 2020 Chinese guidelines for the diagnosis and treatment of systemic lupus erythematosus, the use of SLEDAI-2000 score combined with PGA to evaluate patient's overall disease activity is recommended. ${ }^{[19]}$ In this registry, SLEDAI, PGA, and SLICC damage index were used to assess disease activity and organ damage at the entry and during each follow-up visit. Since the CSTAR registry is initiated, 9486 patients with more than one follow-up visit, 5442 patients have one follow-up, 2042 patients have two visits, 1008 have three visits, 529 patients have four follow-up visits, and 465 patients have more than five follow-up visits are recorded. These longitudinal data can provide strong support for future studies.

In conclusion, during the past 10 years since the CSTAR registry was initiated, more than 25,000 Chinese SLE patients were registered and approximately 10,000 of them were followed in the registry. In this study, we outlined the major demographic and clinical characteristics of Chinese SLE patients. As the largest Chinese SLE patient cohort, the CSTAR registry would provide support for future studies and international collaborations.

Declarations and Funding

None

Conflict of Interest

The authors declare no conflicts of interest.

Ethical Statement

There are no ethical related issues to this article.

Informed Consent

Informed consents have been obtained. 


\section{References}

[1] Rees F, Doherty M, Grainge MJ, et al. The Worldwide Incidence and Prevalence of Systemic Lupus Erythematosus: A Systematic Review of Epidemiological Studies. Rheumatology. 2017;56(11):1945-1961. doi: 10.1093/rheumatology/kex260.

[2] Zeng QY, Chen R, Darmawan J, et al. Rheumatic Diseases in China. Arthritis Res Ther. 2008;10:R17-R27. doi: 10.1186/ar2368.

[3] Merrell M, Shulman LE. Determination of Prognosis in Chronic Disease, Illustrated by Systemic Lupus Erythematosus. J Chronic Dis. 1955;1(1):1-32. doi: 10.1016/0021-9681(55) 90018-7.

[4] Borchers AT, Keen CL, Shoenfeld $Y$, et al. Surviving the Butterfly and the Wolf: Mortality Trends in Systemic Lupus Erythematosus. Autoimmun Rev. 2004;3(6):423-453. doi: 10.1016/j.autrev.2004.04.002

[5] Tektonidou MG, Lewandowski LB, Hu J, et al. Survival in Adults and Children with Systemic Lupus Erythematosus: A Systematic Review and Bayesian Meta-Analysis of Studies from 1950 to 2016. Ann Rheum Dis. 2017;76(12):2009-2016. doi: 10.1136/annrheumdis-2017-211663.

[6] Gladman D, Ginzler E, Goldsmith C, et al. Systemic Lupus International Collaborative Clinics: Development of a Damage Index in Systemic Lupus Erythematosus. J Rheumatol. 1992;19:1820-1821.

[7] Rasmussen A, Sevier S, Kelly JA, et al. The Lupus Family Registry and Repository. Rheumatology (Oxford). 2011;50:47-59.

[8] Reveille JD, Moulds JM, Ahn C, et al. Systemic Lupus Erythematosus in Three Ethnic Groups: I. The Effects of HLA Class II, C4, and CR1 Alleles, Socioeconomic Factors, and Ethnicity at Disease Onset. LUMINA Study Group. Lupus in Minority Populations, Nature Versus Nurture. Arthritis Rheum. 1998;41:1161-1172.

[9] Cervera R, Khamashta MA, Font J, et al. Systemic Lupus Erythematosus: Clinical and Immunologic Patterns of Disease Expression in a Cohort of 1,000 Patients. Medicine (Baltimore). 1993;72:113-124.

[10] Petri M. The Effect of Race on the Presentation and Course of SLE in the United States. Arthritis Rheum. 1997;40:S162[abstract].

[11] Alarcón GS, McGwin G Jr, Petri M, et al. Baseline Characteristics of a Multiethnic Lupus Cohort: PROFILE. Lupus. 2002;11:95-101.

[12] Pons-Estel BA, Catoggio LJ, Cardiel MH, et al. The GLADEL Multinational Latin American Prospective Inception Cohort of 1,214 Patients with Systemic Lupus Erythematosus: Ethnic and Disease Heterogeneity Among "Hispanics". Medicine
(Baltimore). 2004;83:1-17.

[13] Hochberg MC. Updating the American College of Rheumatology Revised Criteria for the Classification of Systemic Lupus Erythematosus. Arthritis Rheum. 1997;40(9):1725.

[14] Dahlström Ö, Sjöwall C. The Diagnostic Accuracies of the 2012 SLICC Criteria and the Proposed EULAR/ACR Criteria for Systemic Lupus Erythematosus Classification are Comparable. Lupus. 2019;28(6):778-782. doi: 10.1177/0961203319846388.

[15] Aringer M, Costenbader K, Daikh D, et al. 2019 European League Against Rheumatism/American College of Rheumatology Classification Criteria for Systemic Lupus Erythematosus. Ann Rheum Dis. 2019;78(9):1151-1159. doi: 10.1136/annrheumdis-2018-214819.

[16] Gladman DD, Ibanez D, Urowitz MB. Systemic Lupus Erythematosus Disease Activity Index 2000. J Rheumatol.

[17] D'Cruz DP, Khamashta MA, Hughes GRV. Systemic Lupus Erythematosus. Lancet. 2007;369:587-596.

[18] Mok CC, MakA, Chu WP, et al. Long-Term Survival of Southern Chinese Patients with Systemic Lupus Erythematosus: A Prospective Study of All Age-Groups. Medicine (Baltimore). 2005;84:218-224.

[19] To CH, Mok CC, Tang SS, et al. Prognostically Distinct Clinical Patterns of Systemic Lupus Erythematosus Identified by Cluster Analysis. Lupus 2009;18:1267-1275.

[20] Li MT, Zhao, Zhang ZY, et al. 2020 Chinese Guidelines for the Diagnosis and Treatment of Systemic Lupus Erythematosu. Rheumatol Immunol Res. 2020;1(1):5-25. doi: 10.2478/rir2020-0009.

[21] Wu J, Ma J, Bao C, et al. Pregnancy Outcomes Among Chinese Women with and without Systemic Lupus Erythematosus: A Retrospective Cohort Study. BMJ Open. 2018;8(4):e020909. doi:10.1136/bmjopen-2017-020909.

[22] Wei Q, Ouyang $Y$, Zeng W, et al. Pregnancy Complicating Systemic Lupus Erythematosus: A Series of 86 Cases. Arch Gynecol Obstet. 2011;284(5):1067-1071. doi: 10.1007/ s00404-010-1786-5.

[23] Lazzaroni MG, Dall'Ara F, Fredi M, et al. A Comprehensive Review of the Clinical Approach to Pregnancy and Systemic Lupus Erythematosus. J Autoimmun. 2016;74:106-117. doi: 10.1016/j.jaut.2016.06.016.

[24] Li M, Zhang W, Leng X, et al. Chinese SLE Treatment and Research Group (CSTAR) Registry: I. Major Clinical Characteristics of Chinese Patients with Systemic Lupus Erythematosus. Lupus. 2013;22:1192-1199. 